\section{SAT0449 SEX, METABOLIC CO-MORBIDITIES AND LINE OF THERAPY PREDICT TNF-INHIBITOR THERAPY PERSISTENCE IN PSORIATIC ARTHRITIS: A RETROSPECTIVE COHORT STUDY}

W. Ye ${ }^{1}$, T. Guruparan ${ }^{1}$, C. Stober ${ }^{2}$, E.E.P. Htut ${ }^{2}$, D.R. Jadon ${ }^{2} .{ }^{1}$ School of Clinical Medicine, University of Cambridge; ${ }^{2}$ Department of Rheumatology, Cambridge University Hospitals, Cambridge, United Kingdom

Background: Although tumour necrosis factor-alpha inhibitor (TNFi) therapy has proven efficacy in the management of psoriatic arthritis (PsA), relatively little is known about predictors of TNFi persistence. Such knowledge would assist the application of stratified medicine.

Objectives: To determine baseline clinical characteristics associated with TNFi persistence in patients receiving their first-line agent, and to compare TNFi persistence in first versus second-line users.

Methods: A retrospective cohort study was performed of all patients with PsA attending a single-centre between 2003-2016. Demographic, clinical and laboratory characteristics were compared with TNFi persistence, using KaplanMeier survival analysis and multivariable Cox proportional hazards models.

Results: A total of 188 PsA cases had used TNFi therapy as first-line over a period spanning 7,620 person months: $46 \%$ male; mean age at TNFi initiation 47.27 (SD 11.36) years; median disease duration at initiation 11 (IQR 7,16) years. Etanercept was used by 102 and adalimumab by 86 . Concomitant DMARDs were used by $121 / 186(65 \%)$ and $87 / 188(46 \%)$ had metabolic co-morbidities (hypertension, dyslipidaemia, type-2 diabetes, obesity). TNFi therapy was terminated in $65 / 188$ (35\%) of cases (35\% due to primary inefficacy, $22 \%$ secondary inefficacy, $43 \%$ adverse events), with a median duration of TNFi persistence of 26.5 (IQR $10.5,62.0)$ months. Multivariable Cox proportional hazards modelling found the following parameters at TNFi initiation to be associated with shorter (poorer) TNFi persistence in first line users: female sex (hazard ratio, HR 2.57; $95 \% \mathrm{Cl}$ $1.26,5.24 ; p=0.01$ ); presence of metabolic co-morbidity (HR 2.65; $95 \% \mathrm{Cl} 1.24$, $5.69 ; \mathrm{p}=0.01$ ); with a non-significant statistical trend towards younger age at $\mathrm{TNFi}$ initiation (HR 0.94; $95 \% \mathrm{Cl} 0.88,1.00 ; \mathrm{p}=0.06$ ) and older age at PsA onset (HR $1.05 ; 95 \% \mathrm{Cl} 0.99,1.12 ; \mathrm{p}=0.08$ ). Parameters not statistically associated with TNFi persistence included: choice of TNFi agent (adalimumab vs. etanercept), concomitant DMARD/methotrexate use, tender/swollen joint counts, patient global assessment (PGA) of disease activity, CRP, ESR and disease duration. Of 32 cases proceeding to a second TNFi (19 adalimumab, 13 etanercept), persistence was $14 / 32(44 \%)$ over 954 person months. TNFi failure was two-fold more likely in second versus first-line users ( $\mathrm{HR} 2.02 ; 95 \% \mathrm{Cl} 1.20,3.42 ; \mathrm{p}=0.009$ ) [Figure $1]$, with no significant contribution from other co-variables.

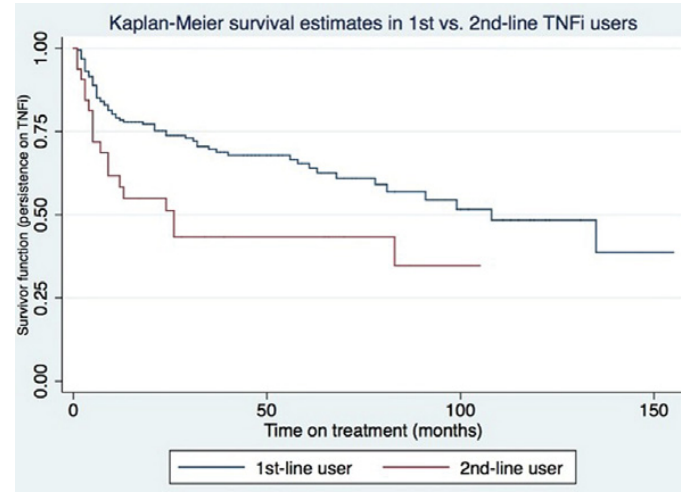

Conclusions: Patients with PsA who are female and have metabolic comorbidities appear to be more likely to fail first-line TNFi therapy. Contrary to observations in rheumatoid arthritis, and somewhat challenging the few studies in PsA, choice of TNFi agent (humanised monoclonal vs. chimeric), concomitant methotrexate use, acute-phase response and joint counts prior to TNFi initiation did not influence TNFi persistence. Although TNFi failure was more likely in second-line users, a significant proportion of PsA cases responded to second-line TNFi therapy, advocating this strategy in refractory cases.

Acknowledgements: TG and WY contributed equally to this study.

Disclosure of Interest: None declared

DOI: 10.1136/annrheumdis-2017-eular.5178

\section{SAT0450 SILENT AXIAL DISEASE IN THE RUSSIAN COHORT OF EARLY PERIFERAL PSORIATIC ARTHRITIS PATIENTS}

E.E. Gubar, E.Y. Loginova, A.V. Smirnov, S.I. Glukhova, T.V. Korotaeva. V. A. Nasonova Research Institute of Rheumatology, Moscow, Russian Federation

Background: Axial involvement in early psoriatic arthritis (ePsA) patients (pts) is often asymptomatic, poorly diagnosed and has not been studied properly. Magnetic resonance imaging (MRI) of sacroiliac joints (SIJs) helps to better define spinal involvement in ePsA.

Objectives: to study the prevalence of axial involvement in peripheral ePsA pts. Methods: 89 pts (M/F-42 /47) with peripheral ePsA according to CASPAR criteria were included; mean age $36.5 \pm 10.9$ yrs, disease duration $12.1 \pm 10.1$ mo., disease activity index (DAS) $5.2 \pm 2.8$, C-RP 16.1 [6.6; 31.0$] \mathrm{mg} / \mathrm{l}$, ESR $22.5 \pm 19.2$ $\mathrm{mm} / \mathrm{h}$. All patients were evaluated for the presence of inflammatory back pain (IBP) by ASAS criteria. In pts having IBP disease activity was also measured according to BASDAI. The examination included X-ray of sacroiliac joints (SIJs) (pelvic radiograph), HLA B27 antigen, MRI of SIJs was performed in 79 pts, both with and without IBP, on Signa Ovation 0,35T. Bone marrow edema on MRI (STIR), considered as active MRI sacroilitis (MRI-SI), was evaluated by an independent reader. Radiographic sacroilitis (R-SI) was defined when 2 grade changes in at least one SIJ appear, and included definite radiographic SI (dR-SI) and "non-definite" radiographic SI (ndR-SI). dR-SI was determined according to New York criteria (unilateral grade $\geq 3$ or bilateral grade $\geq 2$ ). 2 grade changes in only one SIJ were qualified as ndR-SI.

Results: IBP was found in 58 out of $89(65.1 \%)$ pts, $35(60.3 \%)$ of them had short-term (episodic) IBP, and $23(39.7 \%)$ pts had long-term IBP. MRI-SI was observed in 28 out of $79(35.4 \%)$ pts. R-SI was determined in 42 out of 89 $(47.2 \%)$ pts, while dR-SI was found in 27 out of $89(30.3 \%)$ pts, the remaining $15(16.9 \%)$ pts had ndR-SI. 34 (38. 2\%) pts were HLA B27 positive. In pts having IBP disease activity according to BASDAI was $4.5 \pm 1.6$. Correlation has been detected between MRI-SI and IBP: among the group of pts having MRI-SI IBP was observed in $92.9 \%$ cases while out of the group of patients without MRI-SI in $54.9 \%$ cases $(p=0.0002)$. An association was found between MRI-SI and long-term IBP $(p=0.003)$ as well as between MRI-SI and short-term IBP $(p=0.006)$. Moderate correlation has been detected between the presence of $\mathrm{dR}-\mathrm{SI}$ and IBP $(\mathrm{p}=0.038)$. It's worth noting that among the 26 pts having dR-SI, $5(19.2 \%)$ pts never had IBP before. And among the 15 pts having ndR-SI, $6(40.0 \%)$ pts never had IBP before. No association was found between the presence of MRI-SI/R-SI/dR-SI and HLA B27 status.

Conclusions: in the Russian cohort of early peripheral psoriatic arthritis pts, careful examination quite often revealed high prevalence of axial involvement: $65 \%$ of pts had IBP (moreover, more than half of them $(60 \%)$ had short-term IBP), $35 \%$ of pts had MRI-SI, half of the pts had R-SI, one third of the pts had definite R-SI. A significant number of patients (40\%) developed 2 grade changes in one SIJ without previous IBP. An association was found between IBP and SI revealed by any of the visualization methods used. No association has been detected between the presence of MRI-SI or R-SI and HLA B27 status. These data indicate that in peripheral ePsA pts axial involvement is often asymptomatic and is poorly diagnosed. Careful identification of IBP together with MRI of SIJs will help to better define spinal involvement.

Disclosure of Interest: None declared

DOI: 10.1136/annrheumdis-2017-eular.3044

\section{SAT0451 THE ONE-YEAR RADIOGRAPHIC PROGRESSION AND MINIMAL DISEASE ACTIVITY IN EARLY PSORIATIC ARTHRITIS PATIENTS TREATED ACCORDING TO TREAT-TO-TARGET STRATEGY (RESULTS OF AN ONGOING OPEN-LABEL REMARCA STUDY)}

E. Loginova, T. Korotaeva, A. Smirnov, A. Koltakova, D. Karateev. V.A.Nasonova Research Institute of Rheumatology, Moscow, Russian Federation

Background: Treat-to-target (T2T) strategy has benefit in early psoriatic arthritis (EPsA) treatment. Its influence on radiographic progression has limited data.

Objectives: to investigate radiographic outcomes and minimal disease activity (MDA) after 1-year of T2T strategy in patients with EPsA.

Methods: 40 (M-18/F-22) DMARD-naïve pts with active EPsA, according to the CASPAR criteria, mean age $38.4 \pm 11.1$ yrs., PsA duration $11.9 \pm 10.6$ months, psoriasis duration $73.8+84.6$ months, median DAS $3.8[3.2 ; 4.7]$ from the open-label REMARCA study were included. At baseline all pts were treated by Methotrexate (MTX) subcutaneous (s/c) $20-25 \mathrm{mg} /$ week. Pts that still had medium or high activity after 3-6 months were treated by combination therapy MTX+ biologic therapy (BT) - anti-TNF or Ustekinumab $(n=21)$. By the end of the study, 19 pts were treated by MTX-monotherapy. At baseline and after 1-yr. of treatment PsA activity index and digital radiographs of hands and feet were performed. All images were scored according to Sharp/van der Heijde (Sh.-v.d. H) method by a blinded musculoskeletal radiologist. Median total score (TS Sh.-v.d. $H)=$ total erosion score (TES) + total narrowing score (TNS), the proportion of pts who reached MDA, M $\pm S D$, Me [Q75; Q50], W-test, U-test, (\%) were calculated. All $p<0.05$ were considered to indicate statistical significance.

Results: At baseline, 23 out of 40 pts (57\%) had erosion. By 1 yr., the number of pts with erosion increased up to 26 pts $(65 \%)$. The median TS Sh.-v.d. H significantly increased from 91.5 [72-108.5] to 91.5 [73.5-111.5] (W-test, $p<0.01$ ), TES from $2[0-4.5]$ to $2.5[0-5]$ (W-test, $p<0.05)$ and TNS from 85 [69-105] to 87 [71.5-107] (W-test, $p<0.01$ ). At $1 \mathrm{yr}$. of therapy there was no significant difference between the treatment groups in the value of TS Sh.-v.d. H (W-test $p>0.05)$. 25 of pts $(62.5 \%)$ had reached MDA by $1 \mathrm{yr}$. In pts who did not reach MDA $(n=15)$ by $1 \mathrm{yr}$. TES was significantly higher at baseline compare to those who reached MDA: 3 [2-9] and 0 [0-3] accordingly (U-test, $p<0.05$ ). In the group of pts who did not reach MDA 1 yr. progression was significantly higher (table).

In 29 out of 40 pts $(72.5 \%)$ there was no X-ray progression considering both erosion and joint space narrowing. 13 of them $(45 \%)$ were treated by MTX and 16 pts $(55 \%)$ by MTX+BT. Negative X-ray progression was found in 11 out of 40 pts (27.5\%): 6 of them (54.5\%) were treated by MTX and 5 pts (45.5\%) by MTX+BT. 